\title{
AVALIAÇÃO DO TRATAMENTO DA HÉRNIA INGUINAL SOB ANESTESIA LOCAL E SEDAÇÃO EM 1560 PACIENTES
}

\author{
OUTCOME OF GROIN HERNIA REPAIR UNDER LOCAL ANESTHESIA AND SEDATION \\ IN 1560 PATIENTS
}

\author{
José Carlos de Rezende Pereira, ACBC-RJ'1; José Carlos Vieira Trugilho,TCBC-RJ²; \\ Jose Marcus Raso Eulálio,TCBC-RJ ${ }^{3}$; Nélson Jamel TCBC-RJ ${ }^{4}$
}

\begin{abstract}
RESUMO: Objetivo: Analisar a aplicabilidade da anestesia local da região inguinal no tratamento da hérnia inguinal. Método: Foram estudados os dados de 1560 pacientes submetidos ao tratamento operatório para cura de hérnia inguinal, entre maio de 1996 e dezembro de 2003, pela técnica de Lichtenstein, sob anestesia local da região inguinal associada à sedação. Foram analisados so seguintes dados: idade,sexo,índice de massa corporal, número de recidivas, as intercorrências pré e pós-operatórias, tempo de permanência hospitalar. Os tipos de hérnia foram classificados segundo Nyhus. Resultados: Todas as operações foram concluídas sob a anestesia local e sedação não sendo necessário nenhuma complementação. As complicações pós-operatórias ocorreram em $7,16 \%$ dos pacientes, nenhum deles necessitou de reinternação. Conclusão: A anestesia loco-regional da região inguinal na totalidade dos casos produziu conforto para os pacientes e boas condições de operabilidade para os cirurgiões (Rev. Col. Bras. Cir. 2006; 33(6): 375-379).
\end{abstract}

Descritores: Hérnia inguinal; Anestesia local; Procedimentos Cirúrgicos Operatórios

\section{INTRODUÇÃO}

Hérnia em latim significa ruptura ou rasgo e foi primeiramente descrita 1500 anos antes de Cristo, sendo Celso, no primeiro século da era cristã, o precursor do tratamento cirúrgico da hérnia ${ }^{1}$. Edoardo Bassini ${ }^{2}$ em 1884, iniciou uma nova etapa na cirurgia da hérnia inguinal ao propor o reforço da parede posterior para correção da hérnia.

Lichtenstein $^{3}$ em 1970, foi um dos primeiros a defender a anestesia local e a cirurgia ambulatorial para o tratamento da hérnia inguinal e em 1992 esse procedimento já era proposto em nosso país ${ }^{4}$. Em 1969 Falci relatou a aplicação, com sucesso, em 100 pacientes da técnica sem tensão para a correção da hérnia inguinal ${ }^{5}$. Posteriormente uma técnica semelhante foi amplamente divulgada ${ }^{6}$.

O presente estudo tem como objetivo aferir os resultados obtidos no tratamento de 1560 pacientes submetidos à cirurgia para reparo da hérnia inguinal, realizadas no Serviço de Cirurgia do Hospital Municipal Carlos Tortelly, da Fundação Municipal de Saúde de Niterói (FMS), em regime de Hospital Dia, com a utilização do método preconizado por Lichtenstein ${ }^{6}$ sob anestesia local e sedação.

\section{MÉTODOS}

Foram analisados os pacientes submetidos ao tratamento cirúrgico da hérnia inguinal unilateral, entre janeiro de
1996 e dezembro de 2003, sob anestesia loco-regional e sedação.

Para o registro de dados, foi elaborada uma ficha modelo onde foram coletados os seguintes tópicos: idade, sexo, peso, altura, tipo de hérnia inguinal segundo a classificação de Nyhus ${ }^{7}$, o índice de massa corporal (IMC), número de recidivas, intercorrências per-operatórias, pós-operatórias e tempo permanência hospitalar.

No pré-operatório, os pacientes foram encaminhados para avaliação clínica e os exames complementares préoperatórios pedidos de acordo com critérios preconizados pelo Colégio Brasileiro de Cirurgiões ${ }^{8}$.

Todos os pacientes foram orientados previamente sobre: a alta precoce, a necessidade de ter um acompanhante e o meio de transporte..

\section{Técnica Anestésica}

Após a internação o paciente era entrevistado pelo anestesiologista, que participaria do ato operatório, para esclarecimentos sobre o bloqueio anestésico. $\mathrm{Na}$ sala de cirurgia, após venóclise periférica em membro superior e monitoração com cardioscópio, oxímetro de pulso e mensuração não invasiva da tensão arterial, era feito midazolan $10 \mathrm{mg}$ venoso e meperidina $50 \mathrm{mg}$ por via intramuscular. Com isso tornamos o paciente bastante cooperativo quando da realização da infiltração anestésica.

\footnotetext{
1. Chefe do Serviço de Cirurgia do Centro Previdenciário de Niterói e Coordenador de Cirurgia do Programa Médico de Família de Niterói; Mestrando do Programa Pós-graduação em Cirurgia da Universidade Federal do Rio de Janeiro.

2. Professor Adjunto da Universidade Federal Fluminense e Chefe do Departamento de Cirurgia da Universidade Federal Fluminense.

3. Professor Adjunto do Departamento de Cirurgia da Faculdade de Medicina- UFRJ.

4. Professor Titular do Departamento de Cirurgia da Faculdade de Medicina- UFRJ.

Recebido em 19/06/06

Aceito para publicação em 18/07/06

Conflito de interesses: nenhum

Fonte de financiamento: nenhuma

Trabalho realizado no Serviço de Cirurgia Ambulatorial do Hospital Municipal Carlos Tortelly.
} 
A solução anestésica para o bloqueio da região inguinal foi preparada com: $20 \mathrm{ml}$ de lidocaina à $2 \%, 20 \mathrm{ml}$ de bupivacaina com vasoconstrictor à $0,5 \%, 40 \mathrm{ml}$ de solução salina à $0,9 \%$ e $4 \mathrm{ml}$ de bicarbonato de sódio à $8,4 \%$.

Para a realização da infiltração da solução anestésica na região inguinal consideramos os seguintes pontos de reparo : Ponto1 - espinha ilíaca antero-superior e Ponto 2- tubérculo púbico (Figura 1).

A infiltração profunda de $15 \mathrm{ml}$ da solução anestésica era feita, em ângulo reto com a pele, junto à espinha ilíaca ântero-superior ( Figura 2). Do ponto 1 ao umbigo e ao tubérculo púbico infiltramos cerca de $20 \mathrm{ml}$ de anestésico nos espaços intradérmicos, subcutâneo e muscular (Figuras 3 e 4). A seguir, com $10 \mathrm{ml}$ de solução anestésica, infiltrava-se profundamente margeando o ramo superior do púbis, em cada lado do cordão espermático, dentro da fixação do músculo reto ao púbis e o espaço de Retzius (Figura 5). Nos pacientes obesos fizemos um leque de anestésico no tecido celular subcutâneo (Figura 6). Não foram feitas tentativas para infiltrar o plexo simpático, do nervo gênitofemoral ou o peritônio no anel inguinal profundo, pois estas manobras podem ser desastrosas, podendo provocar hematomas que prejudicam o ato cirúrgico. Quando necessário, infiltrações sob visão direta são feitas pelo cirurgião sem risco de lesar estruturas adjacentes.

Para a correção das hérnias utilizamos em todos os pacientes a técnica descrita por Lichteinstein ${ }^{9}$. As hérnias bilaterais foram operadas em atos cirúrgicos distintos.

\section{RESULTADOS}

Foram operados 1560 pacientes sendo 1353 (86,7\%) do sexo masculino e 207 (13,3\%) do sexo feminino. A média de idade foi de 47 anos, com a idade mínima de 15 e a idade máxima de 90 anos. 19,6\% dos pacientes encontravam-se entre 61 e 70 anos e $54,8 \%$ entre 41 e 70 anos. (Tabela 1 ).

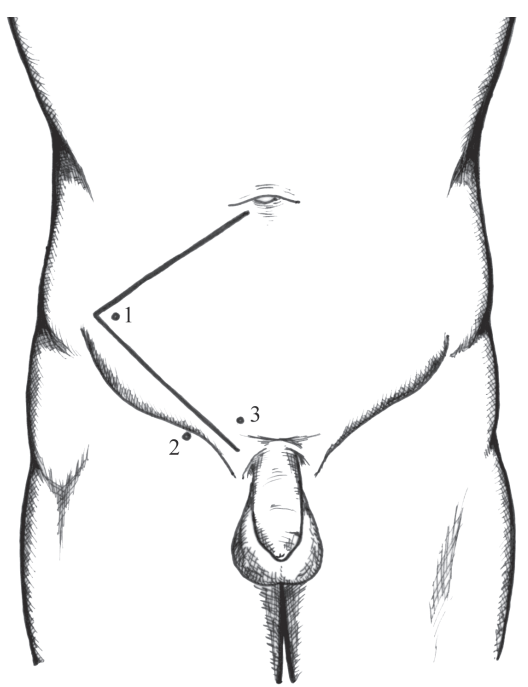

Figura 01

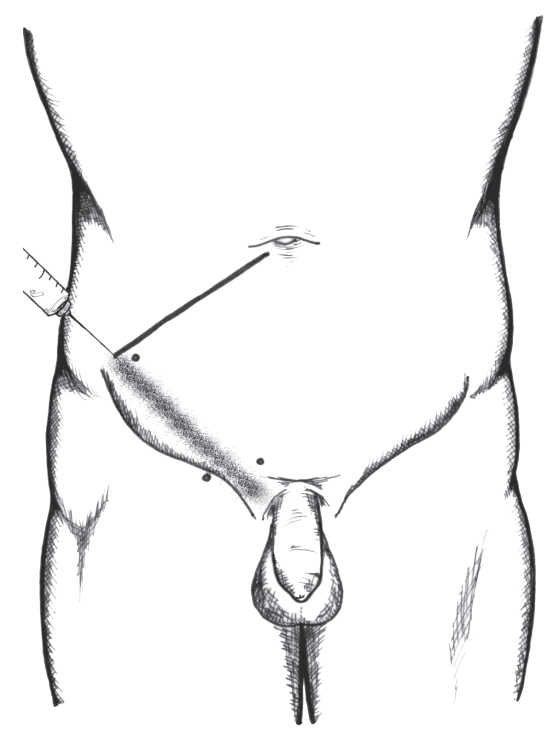

Figura 04

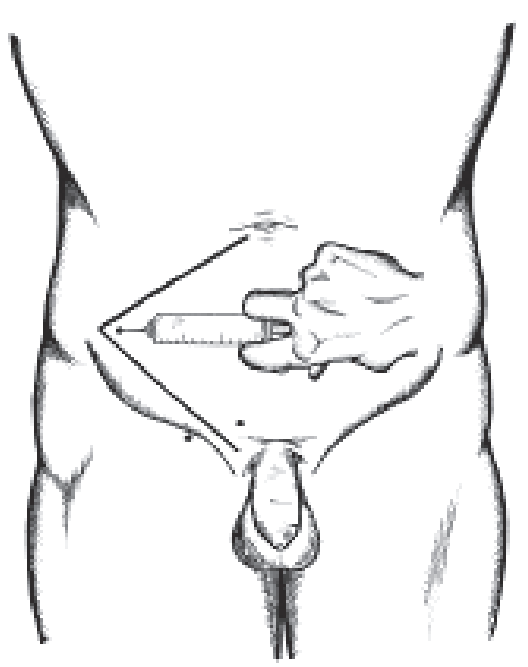

Figura 02

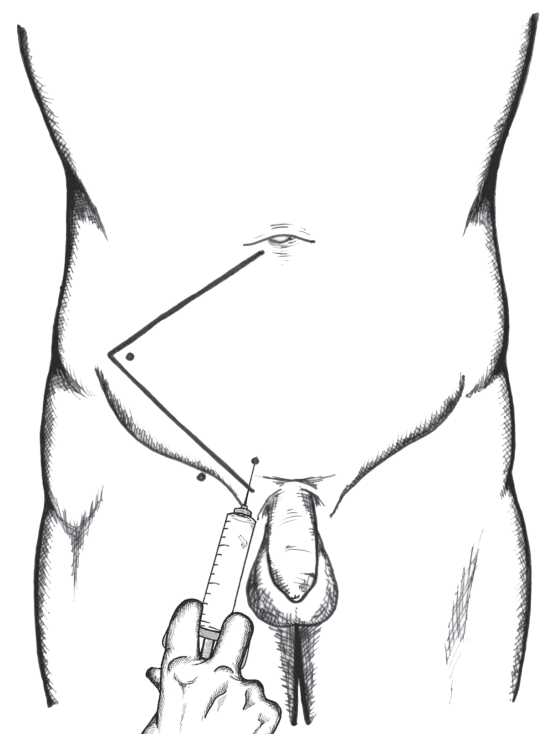

Figura 05

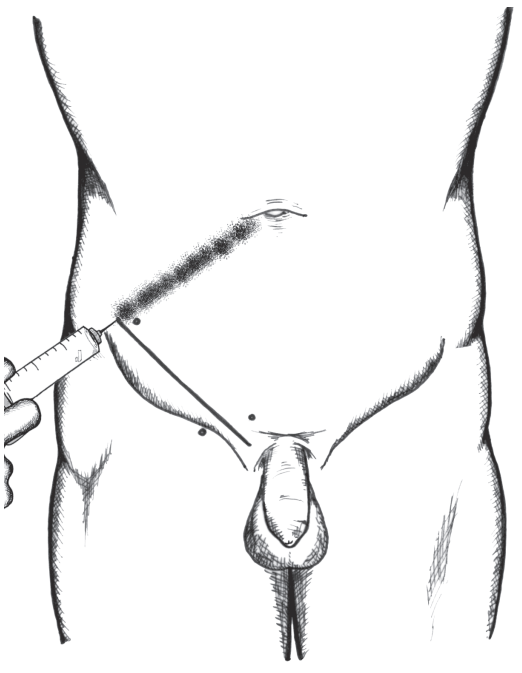

Figura 03

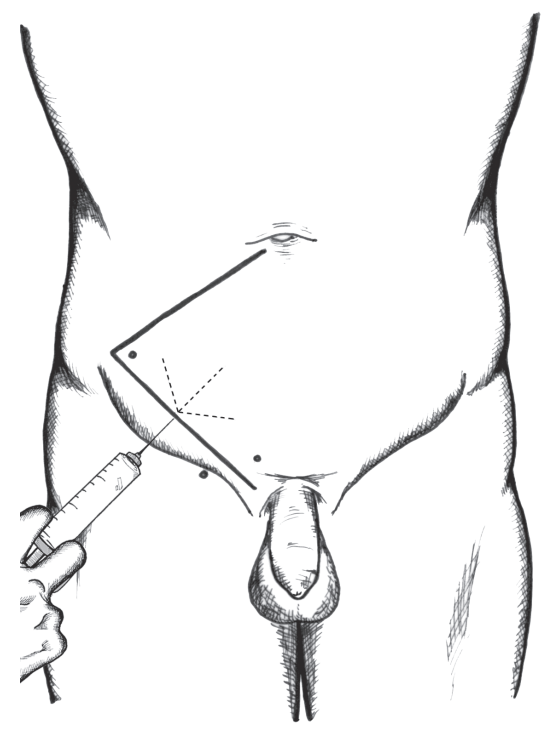

Figura 06 


\section{Tabela 1}

\begin{tabular}{lc}
\hline Idade/Anos & Pacientes \\
\hline $\mathbf{1 0}$ a 20 & 130 \\
$\mathbf{2 1}$ a 30 & 214 \\
$\mathbf{3 1}$ a 40 & 203 \\
$\mathbf{4 1}$ a $\mathbf{5 0}$ & 281 \\
$\mathbf{5 1}$ a $\mathbf{6 0}$ & 269 \\
$\mathbf{6 1}$ a 70 & 306 \\
$\mathbf{7 1}$ a 80 & 120 \\
$\mathbf{8 1}$ a 90 & 37 \\
Número de pacientes & 1560 \\
Média & 47,08 \\
Mínima & 15 anos \\
Máxima & 90 anos \\
\hline
\end{tabular}

Tabela 2

\begin{tabular}{lc}
\hline IMC & Pacientes/Percentagem \\
\hline Até 18,5\% & $17(1,1 \%)$ \\
De 18,5\% A 20\% & $87(5,6 \%)$ \\
De 20\% A 25\% & $608(38,9 \%)$ \\
De 25\% A 30\% & $761(48,8 \%)$ \\
Acima de 30\% & $87(5,6 \%)$ \\
Média & 24,67 \\
Desvio Padrão & 2,82 \\
\hline
\end{tabular}

Tabela 3

\begin{tabular}{lrl}
\hline NYHUS & Pacientes/Percentagem \\
\hline Tipo 1 & - & \\
Tipo 2 & 867 & $(55,6 \%)$ \\
Tipo 3-A & 399 & $(25,6 \%)$ \\
Tipo 3-B & 233 & $(14,9 \%)$ \\
Tipo 3-C & - & \\
Tipo 4 & 61 & $(3,9 \%)$ \\
Total & 1560 & $(100 \%)$ \\
\hline
\end{tabular}

Em 87 pacientes, correspondentes a 5,6\% do total, o índice de massa corporal (IMC), era superior a 30 (Tabela 2).

As hérnias foram classificadas segundo Nyhus ${ }^{7}$ quanto ao substrato anatômico (Tabela 3). Encontramos 59,1\% de hérnias indiretas, $24,2 \%$ de hérnias diretas e 3,9\% de hérnias recidivadas.

O tempo médio do ato operatório foi de 37 minutos com o mínimo de 21 minutos e o máximo de 80 minutos. Não houve em nossa casuística, nenhuma complicação durante o ato cirúrgico. Não houve necessidade de complementação anestésica em nenhum dos pacientes desta série.

A média de tempo de permanência hospitalar foi de $6,75 \mathrm{~h}$, com o mínimo de 6 h e máximo de $10 \mathrm{~h}$ (Tabela 4).

O hematoma da região inguinal foi a complicação pósoperatória mais freqüente, ocorreu em 3,9\% dos pacientes. $\mathrm{O}$ índice total de complicações foi de 7,16\% (Tabela 5). Não houve complicações pós-operatórias que justificassem re-

\section{Tabela 4}

\begin{tabular}{lcc}
\hline Internação & \multicolumn{2}{c}{ Pacientes/Percentagem } \\
\hline $\mathbf{6 h}$ & 903 & $(57,9 \%)$ \\
$\mathbf{7 h}$ & 184 & $(11,8 \%)$ \\
$\mathbf{8 h}$ & 447 & $(28,7 \%)$ \\
$\mathbf{9 h}$ & 09 & $(0,6 \%)$ \\
$\mathbf{1 0 h}$ & 17 & $(1,1 \%)$ \\
Acima de 10h & - & \\
Média & \multicolumn{2}{c}{6,75} \\
Desvio Padrão & \multicolumn{2}{c}{0,97} \\
\hline
\end{tabular}

Tabela 5

\begin{tabular}{lrl}
\hline Complicações & Pacientes/Percentagens \\
\hline Equimose & 32 & $(2,1 \%)$ \\
Hematoma Inguinal & 60 & $(3,9 \%)$ \\
Hematoma escrotal & 12 & $(0,7 \%)$ \\
Infecção de parede & 6 & $(0,4 \%)$ \\
Dor crônica & 1 & $(0,06 \%)$ \\
Total & 111 & $(7,16 \%)$ \\
\hline
\end{tabular}

internação ou re-intervenção cirúrgica. Não houve recidiva durante o $1^{\circ}$ ano de pós-operatório.

\section{DISCUSSÃO}

A partir de 1970 , com a retomada da cirurgia ambulatorial, passou a se buscar alternativas para os procedimentos anestésicos que viabilizassem esse tratamento ${ }^{10}$. No nosso país alguns autores enfatizam a utilização deste tipo de bloqueio anestésico, associado à sedação, para a maioria dos pacientes que serão submetidos à cura cirúrgica de hérnias inguinais ${ }^{11-13}$

Pacientes com alergia ao anestésico local ou condições clínicas que contra-indiquem a anestesia raquidiana ou peridural justificariam o uso regular da anestesia geral, embora inúmeros trabalhos defendam o uso da anestesia local como a melhor opção para o tratamento da hérnia inguinal ${ }^{14-16}$. A anestesia local é indiscutivelmente a menos invasiva e com a menor resposta inflamatória, sendo assim a mais segura de todas as técnicas anestésicas para cirurgia da hérnia inguinal, além da possibilidade de produzir alívio relativamente prolongado da dor pós-operatória, não precisamos administrar analgésicos no pós-operatório imediato até a alta hospitalar em nenhum dos pacientes. Além disso a anestesia loco-regional da região inguinal acompanhada de sedação, na totalidade dos casos, produziu conforto para os pacientes e boas condições de operabilidade para os cirurgiões.

Em estudo com 189 pacientes acima de 80 anos submetidos à cirurgia de hérnia sob anestesia local, demonstrouse que a idade não é um fator limitante para o procedimento proposto ${ }^{17}, 157$ pacientes com mais de 70 anos cuja evolução 
pós-operatória foi semelhante à dos pacientes com faixa etária mais baixa.

Este tipo de bloqueio anestésico permitiu que fossem operados também os 87 pacientes obesos que apresentavam IMC acima de 30. Não houve dificuldade na execução da operação de hérnia com anestesia loco-regional em nenhum dos tipos da classificação de Nyhus ${ }^{7}$ presentes em nossa série.

$\mathrm{Na}$ evolução do proposto por Lichtenstein ${ }^{6}$, os autores experimentaram resultados melhores em relação às contra indicações formalizadas pelo trabalho original. Assim pacientes com doenças sistêmicas, mesmo as cárdio-pulmonares graves, e pacientes portadores de hérnia inguinal recidivada puderam se beneficiar do reparo sem tensão sob anestesia local ${ }^{18}$. O baixo custo aliado à ínfima morbidade e inexistente mortalidade foram os fatores preponderantes que encorajaram vários cirurgiões a usarem este procedimento ${ }^{19}$. Ainda assim, a obesidade, as hérnias encarceradas e as ínguinoescrotais continuavam formalmente contra indicadas para o tratamento sob anestesia local, preferindo-se o bloqueio peridural ou a anestesia geral.

Observamos que o tempo de permanência hospitalar tem razão direta com a técnica anestésica empregada, não havendo necessidade de pernoite de nenhum dos pacientes.
Percebemos que os pacientes com grande ansiedade para serem submetidos à anestesia local podiam ser facilmente controlados pela sedação pré-anestésica, que foi também aplicada nos pacientes psiquiátricos. Das contra-indicações preconizadas por Lichtenstein ${ }^{6}$ nenhuma foi fator de limitação para as cirurgias, com exceção dos pacientes abaixo de 12 anos, pois não temos cirurgia pediátrica em nosso serviço. Não registramos nenhum caso de hipersensibilidade ao anestésico local, ocorrência raríssima em todas as séries consultadas. A última contra indicação seria a discrasia sanguínea, e que em nossa opinião estaria contra indicado não para a anestesia local, mas para o ato operatório sob qualquer técnica anestésica, ainda mais em nossa casuística onde todas as cirurgias foram eletivas.

Concluímos que nos pacientes operados em regime ambulatorial a anestesia loco-regional associada à sedação mostrou-se adequada para a realização da hernioplastia inguinal, posto que deu conforto para os pacientes e boas condições de operabilidade para os cirurgiões.

\section{AGRADECIMENTO}

Os autores agradecem ao Sr. Eduardo Peçanha, aluno do Curso de Belas Artes da UFRJ, pela elaboração das figuras 1-6.

\begin{abstract}
Background: This study aimed at showing the feasibility of local-regional anesthesia in inguinal hernia repair using the technique of Lichtenstein. Methods : 1560 patients were operated between May 1996 and December 2003. They were submitted to 1560 surgeries by means of the Lichtenstein technique, operated under local-regional anesthesia in inguinal region. Age, gender, Nyhus classification, and body mass index were analyzed. Results: The post-operative complications had occurred in 7,16\% of the patients. Hospitalization average were 6:00 hours. Conclusions: We concluded that local-regional anesthesia in the inguinal region provides the same level of comfort for patients as well as for surgeons with the advantage of imposing shorter hospital stay.
\end{abstract}

Key words: Hernia, inguinal; Anesthesia, local; Surgical Procedures, Operative.

\section{REFERÊNCIAS}

1. Barreto H. Contribuição ao estudo das bases anatômicas e fisiológicas na cirurgia das hérnias inguinais. Rio de Janeiro: Oficina Gráfica da Universidade do Brasil; 1964.

2. Bassini E, 1997. apud: Abrahamson JR. Hernias. In: Zinner MJ, Schwartz SI, Ellis H, editors. Maingot's abdominal operations. $10^{\text {th }}$ ed. Connecticut: Appleton \& Lange; 1997. p. 479-580.

3. Lichtenstein IL. Hernia repair without disability. $1^{\text {st }}$ ed. St. Louis: Mosby; 1970.

4. Rodrigues Jr AJ, Mittelstaed WEM, Bevilacqua RG, Birolini D. Anestesia por bloqueio de campo no tratamento ambulatorial das hérnias inguinais. Rev Col Bras Cir. 1992;19(5):195-8.

5. Falci F. Use of marlex-mesh in the therapy of inguinal hernia in adults (analysis of 100 operated cases). Hospital. 1969;75(1):14759.

6. Lichtenstein IL, Shulman AG. Ambulatory outpatient hernia surgery. including a new concept, introducing tension-free repair. Int Surg. 1986: 71(1):1-4

7 . Nyhus LM. Individualization of hernia repair: a new era. Surgery. 1993;114(1):1-2
8. Bravo Neto GP, Gonçalves MDC. Pré e pós-operatório. Programa de Auto-avaliação em Cirurgia, Ano I, Fascículo I, Abril 2001

9. Lichtenstein IL, Shulman AG, Amid PK, Montllor MM. The tension-free hernioplasty. Am J Surg. 1989;157(2):188-93.

10. Amado WJ. Anesthesia for groin hernia surgery. Surg Clin North Am. 2003;83(5):1065 -77.

11. Henriques AL, Pezzolo S, Silva GC. Herniorrafia inguinal sob anestesia local. Rev Col Bras Cir. 1997;24(6):405-8.

12. Lima Neto EV, Goldenberg A, Jucá MJ. Resultados imediatos da herniorrafia inguinal com anestesia local associada com sedação. Acta Cir.Bras. 2003; 18(5):478-84.

13. Lopes AG, Souza JCL, Bienik TS, Velozo TS, Nader PA, Cardoso JR. Tratamento da hérnia inguinal com anestesia local. ABCD Arq Bras Cir Dig. 2004; 17(1):26-8.

14. Amid PK, Shulman AG, Lichtenstein IL. Local anesthesia for inguinal hernia repair step-by-step procedure.Ann Surg. 1994;220(6):735-7

15. Armstrong DN, Kingsnorth AN. Local anaesthesia in inguinal herniorrhaphy: influence of dextran and saline solutions on duration of action of bupivacaine. Ann R Coll Surg Engl. 1986;68(4):207-8. 
16. Abdu RA. Ambulatory herniorrhaphy under local anesthesia in a community hospital. Am J Surg .1983;145(3):353-6

17. Spirch S, Martella B, Militello C, Nistri R, De Santis L, Ciardo L, Dalla Vecchia D, Perelda F, Reale F, Sarri C, Volpin E, Terranova O. [Surgery of inguinal and femoral hernia in the elderly]. Chir Ital. 1997;49(4-5):53-5

18. Spallitta SI, Termine G, Zappulla A, Greco V, Compagno GM, Lo Iacono I, Plaja S, Platania P, Ferrara S, Solina G, Moschetti G. [Tension-free hernioplasty in the treatment of inguinal hernia in the adult: our experience with local anesthesia anesthesia and a review of the literature]. Minerva Chir. 1999;54(9):573-89

19. Amid PK, Lichtenstein IL. The Lichtenstein open "tension-free" mesh repair of inguinal hernias. Rozhl Chir. 1995;74(6):296-301.
Como citar este artigo:

Pereira JCR, Trugilho JCV, Eulálio JMR, Jamel, N. Avaliação do tratamento da hérnia inguinal sob anestesia local e sedação em 1560 pacientes. Rev Col Bras Cir [periódico na Internet] 2006;33(6). Disponível em URL: www.scielo.br/rcbc

Endereço para correspondência: José Carlos de Rezende Pereira

Rua Adelino Magalhães, 55

Piratininga

24350-150 - Niterói - RJ

E-mail:jcrezende@globo.com 